\title{
Mathematical models for turbulent round jets based on "ideal" and "lossy" conservation of mass and energy
}

\author{
Fermin Franco ${ }^{1,2, *}$, Yasuhide Fukumoto ${ }^{3}$ \\ ${ }^{1}$ Center for Mathematical Modeling and Data Science, Osaka University, Osaka, Japan \\ ${ }^{2}$ Graduate School of Medicine, Osaka University, Osaka, Japan \\ ${ }^{3}$ Institute of Mathematics for Industry, Kyushu University, Fukuoka, Japan \\ ${ }^{*}$ Corresponding author: franco@sigmath.es.osaka-u.ac.jp
}

\begin{abstract}
We propose mathematical models for turbulent round atomized liquid jets that describe its dynamics in a simple but comprehensive manner with the apex angle of the cone being the main disposable parameter. The basic assumptions are that (i) the jet is statistically stationary and that (ii) it can be approximated by a mixture of two fluids with the phases in local dynamic equilibrium, or so-called locally homogeneous flow (LHF). The models differ in their particular balance of explanatory capability and precision. To derive them we impose partial conservation of the initial mass and energy fluxes, introducing loss factors again as disposable parameters. Depending on each model, the equations admit explicit or implicit analytical solutions or a numerical solution in the discretized model case. The described variables are the the two-phase fluid's composite density and velocity, both as functions of the distance from the nozzle, from which the dynamic pressure is calculated.
\end{abstract}

\section{Keywords}

Mathematical Modeling, Two Phase Fluid, Locally Homogeneous Flow, Statistically Stationary State

\section{Introduction}

The range of applications involving atomizing liquid jets forming two-phase fluid flows is still large. The complexity of the atomizing process, involving numerous physical phenomena and many variables, ranging from the conditions inside the nozzle (or some generating source) to the interaction between the atomization process and the environment into which the jet is penetrating, all account for numerous challenges in physical and mathematical modeling. Notwithstanding, several such models have been attempted to describe different aspects of the jets in this regime. For example, differential equations for a fuel jet's tip penetration distance as a function of time [1, 2, 3]; models for the gas entrainment rate in a full-cone spray [4]; and a one-dimensional model for the induced air velocity in sprays [5]. None of these models is sufficient by itself as explained below.

In this study we propose three original related 1D mathematical models, so-called "energy jet models", for the macroscopic dynamics of a turbulent round jet ensuing from a circular nozzle into a stagnant fluid. This kind of jets serves as a basis for many industrial processes in modern manufacturing industry [6]. An advantage of our models over other analytical 1D models is that the simplest case of the "ideal energy jet" has a single experimentally measurable parameter (the jet half-angle $\theta$ ) while it maintains reasonable predictive power and gives theoretical understanding that allows it to analytically calculate other physical quantities of interest. Moreover, the herein reported other extended models class, the "lossy energy jet" models, apply an energy conservation approach with simple turbulence and energy dissipation models, resulting in increased accuracy. Compared to the present study, past models either lack a description of the density or liquid fraction of the spray, make unrealistic assumptions or introduce parameters unavailable experimentally. There are also widely used numerical models based on turbulence modelling for jets, like the one by [7] used in CFD programs like Star-CD, KIVA-3V, Ansys Fluent, as well as open source codes like OpenFOAM [8]. The present work is mainly concerned with developing an analytical or nearly analytical model with the advantage of producing closed expressions and/or fast calculations.

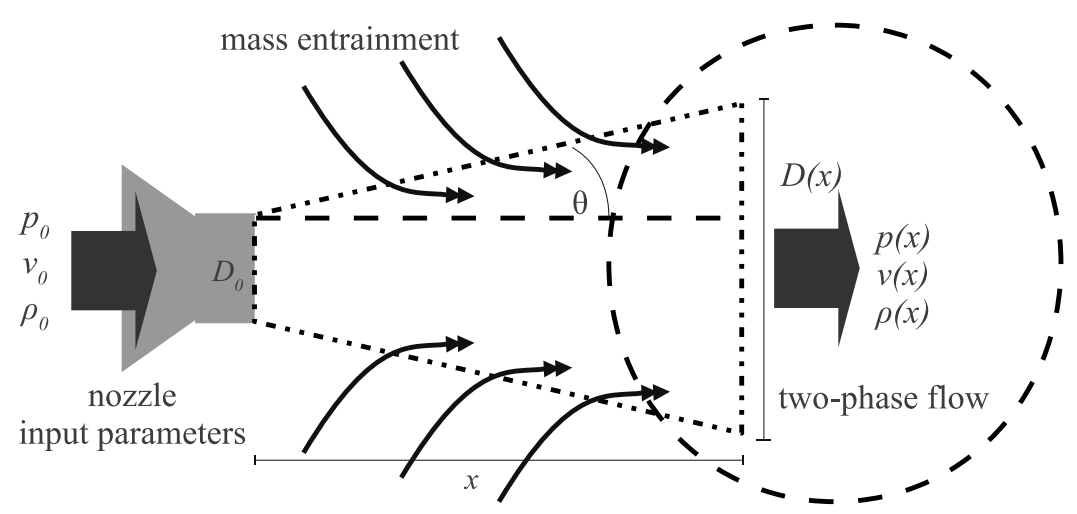

Figure 1. Diagram of the mass entrainment process in a jet and the relevant physical variables. 


\section{The ideal energy jet model}

Consider the statistically stationary state of a full-cone turbulent pressure-atomized liquid jet exiting a single-hole circular nozzle and penetrating into an ambient gas, with constant input liquid gauge pressure and a small conical jet spread angle. We then wish to calculate the dynamical properties of this jet at some axial distance from the nozzle. The relevant variables and parameters are depicted in Figure 1.

\section{Initial power}

The kinetic energy of a flat disc of liquid of infinitesimal width exiting the circular nozzle is

$$
d T=\frac{1}{2} m_{0} v_{0}^{2},
$$

where the mass of the flat disc is $m_{0}=1 / 4 \rho_{0} \pi D_{0}^{2} d z$. Note that $d z=v_{0} d t$. This velocity may be calculated from the input gauge pressure, $p_{0}$, by Bernoulli's theorem, neglecting the dynamic pressure inside the nozzle and assuming that the static pressure is totally converted to the jet's dynamic pressure just outside the nozzle. Thus $p_{0} \approx 1 / 2 \rho_{0} v_{0}^{2}$ implies

$$
v_{0}=\sqrt{\frac{2 p_{0}}{\rho_{0}}} .
$$

Substituting $m_{0}$ and $v_{0}$ from (2) into equation (1) we get

$$
\dot{T}_{0}=\left.\frac{d T}{d t}\right|_{z=0}=\frac{1}{8} \pi \rho_{0} D_{0}^{2} v_{0}^{3}=\frac{1}{2} \pi D_{0}^{2}\left(\frac{p_{0}^{3}}{2 \rho_{0}}\right)^{\frac{1}{2}},
$$

which is the total power (energy flux per unit time) coming out of the nozzle as a result of the input gauge pressure inside the nozzle.

\section{Conservation of power}

We assume that the fluid at a distance $z$ is a two-phase fluid of mixed liquid droplets and gas. The present model has been termed "ideal energy" to distinguish it from the "ideal momentum" developed by the present authors in a previous article $[9,10]$ and the "lossy" models including energy and/or mass loss parameters to be described in sections below. Accordingly, as a first approximation we assume conservation of power, i.e. the energy flux of the two-phase fluid is solely that coming from the original input pressure. The droplets transfer kinetic energy to the initially static gas by drag forces $[11,12]$ and they reach local dynamic equilibrium in such a way that both gas and liquid droplets move at the same speed $v$ inside the jet [13]; this is also the main assumption under the wide class of "Locally Homogeneous Flows" (LHF) $[14,15,16]$. We assume that the latter process occurs so fast immediately outside the nozzle's exit that we may neglect the non-equilibrium zone near the nozzle. The latter assumption is reasonable for high-Reynolds-number pressure-atomized jets, e.g. like the ones used in real life diesel engines [2]. This effectively allows us to treat the two-phase flow as a single fluid with a composite density $\rho$ and a single velocity $v$, both depending on the distance from the nozzle, $z$. Analogous to the calculations in subsection, the kinetic energy of a flat disc of fluid of infinitesimal width at an axial distance $z$ from the nozzle's exit is $d T=1 / 2 v^{2}$, where $m=1 / 4 \rho \pi D^{2} d z$ and $d z=v d t$, and thus

$$
\dot{T}(z)=\left.\frac{d T}{d t}\right|_{z}=\frac{1}{8} \pi \rho D^{2} v^{3},
$$

which is the total power at a cross-section of the two-phase fluid jet at a distance $z$ from the nozzle. Equating (3) and (4) by the idealized assumption of conservation of the energy contained between the two planes $z=0$ and at an axial distance $z$ in steady state, and solving for $v$ we get

$$
v^{3}=\frac{1}{\rho}\left(\frac{D_{0}}{D}\right)^{2} \sqrt{\frac{8 p_{0}^{3}}{\rho_{0}}} .
$$

This can be written in simpler form by using dimensionless units as $\hat{v}^{3}=\hat{\rho}^{-1} \hat{D}^{-2}$, where $v, D$ and $\rho$ have been scaled respectively by $v_{0}, D_{0}$ and $\rho_{0}$ (the quantities at the nozzle's exit) and denoted by a hat symbol.

\section{Volume of gas in the two-phase fluid}

The total fluid volume of the thin disc at the target distance is $d V=d V_{0}+d V_{g}$, where the subscript "g" denotes the quantities related to the "gas", i.e. the total volume $d V$ of the two-phase fluid spray is just the sum of the volume of original quantity of liquid coming out of the nozzle $d V_{0}$ plus the added volume of entrained gas in dynamic equilibrium $d V_{g}$. The volume of liquid is already known since it is just the original volume of liquid. Also, the total volume of the spray at the target distance is straightforward to calculate from the geometry. Then $d V_{g}=d V-d V_{0}$ from where we can calculate a volumetric flow rate of the gas entrainment:

$$
\frac{d V_{g}}{d t}=\frac{1}{4} \pi\left(D^{2} v-D_{0}^{2} v_{0}\right)
$$

Here we can substitute for $v_{0}$ from equation (2) and $D=D_{0}+2 z \tan \theta$. 


\section{Density of the two-phase fluid}

The mean density of the two-phase fluid spray thin-disc element is just the total mass over the total volume:

$$
\rho=\frac{m}{V}=\frac{d m_{0}+d m_{g}}{d V_{0}+d V_{g}}=\frac{\rho_{0} d V_{0}+\rho_{g} d V_{g}}{d V_{0}+d V_{g}}
$$

After substituting $d V_{g}$ from (6), and the initial volume element $d V_{0}$ from analogous calculations, simplifying and solving for $\rho$ we obtain

$$
\rho=\rho_{g}+\frac{D_{0}^{2} v_{0}}{D^{2} v}\left(\rho_{0}-\rho_{g}\right), \quad \text { or } \quad \hat{\rho}=\rho_{*}+\left(1-\rho_{*}\right) / \hat{D}^{2} \hat{v}
$$

the density of the two-phase fluid spray at a distance $z$ from the nozzle. The second equation in (8) is the dimensionless form, where $\rho_{*}=\rho_{g} / \rho_{0}$. Fortunately, it depends on $v$ which makes the dependency circular as we can see from equation (5).

We implicitly assume, by calculating the mean composite density of the two-phase fluid in Equation (7), that the droplets distribution throughout the disc two-phase fluid element does not differ greatly from a uniform distribution. Notice that we approximate the front of the jet by a planar front of equal density, i.e. a "top-hat" radial distribution. In reality this is not true, since the front should be spherical in the first order, and it is then in a spherical shell within the jet's cone that we should consider $\rho$ to be approximately constant, not in a plane. However, for small half-angles $\theta$ and short distances $z$ a plane should suffice as a first order approximation. The same could be said of the front's velocity $v$. Overall, we may take the above considerations as utilizing "top-hat" velocity and density distributions as a first approximation. Note that slicing the spherical jet front with a $z$-normal plane provides a non-constant $\rho$ density distribution in this plane. This distribution should, however, be similar to a two-dimensional Gaussian distribution centered around the $z$-axis, i.e. the jet's "centerline". There are some models $[5,3,17,18,13]$ which apply Gaussian velocity distributions as initial assumptions; however, this calculation will be included in a later work since we anticipate that it would not lead to a major refinement of the axial centreline quantities.

\section{Non-linear equations for the axial velocity and composite density of the spray}

From equations (5) and (8) we identify a system of two non-linear equations with two unknowns, $v$ and $\rho$. We can eliminate $\rho$ to from the system and write in dimensionless form

$$
\rho_{*} \hat{D}^{2} \hat{v}^{3}+\left(1-\rho_{*}\right) \hat{v}^{2}-1=0,
$$

which is a cubic polynomial equation in $\hat{v}$, and which can be solved numerically.

Analogously we can get a non-linear equation for $\hat{\rho}$ eliminating $\hat{v}$ from the same described system and we obtain:

$$
\left(\frac{\hat{\rho}-\rho_{*}}{1-\rho_{*}}\right)^{3}=\frac{\hat{\rho}}{\hat{D}^{4}}
$$

which we can again solve numerically. With both $\hat{\rho}$ and $\hat{v}$ calculated, the dynamic pressure of the two-phase fluid, which accounts for the total pressure at some target axial distance $z$ from the nozzle exit, may be calculated as

$$
\hat{p}=\hat{\rho} \hat{v}^{2},
$$

where $\hat{p}=p / p_{0}$, and $p_{0}=\rho_{0} v_{0}^{2} / 2$ is the initial gauge pressure as in (2).

\section{Energy loss}

In this section we will generalize the above model and, instead of the idealized assumption of perfect conservation of power (energy flux), which is not realistic due to multiple energy loss processes detailed below, we will assume instead that only some proportion of the energy is conserved. Consider a two-phase fluid parcel travelling from the nozzle's exit outward in an axial direction. Physically, we can identify the causes of energy loss of the fluid parcel as at least the following. (i) Droplets' surface energy: taken away by the droplets' surface at jet breakup (due to surface tension). (ii) Droplets' oscillations: the internal elastic motion and deformation of the droplets' surface (which may be approximated by the normal modes of oscillation of a sphere). (iii) Droplets' rotation: produced by some net torque at detachment during breakup. (iv) Turbulence: turbulent motion and energy of created vorticity in the two-phase fluid mainly due to gas entrainment at jet breakup and the turbulent wakes behind the travelling droplets. (v) Escaping droplets: kinetic energy taken away by escaping droplets expelled from the jet's bounded conical geometry by the stochastic breakup process and turbulence (notice this also produces mass loss, although this will be addressed until next section). (vi) Thermal dissipation: some of the kinetic energy is transformed into heat due to the skin friction between the droplets and viscosity.

As stated, it is clear that there are multiple sources of energy loss, but they depend mostly on the following two factors: speed and travel time. Alternatively, travel time at certain speed can be transformed into distance.

Therefore, it is reasonable to assume that the proportion of energy loss, due to at least the above listed factors, should depend on both the speed and travel time of each fluid element (disc). Consider two contiguous fluid elements, i.e. two discs of infinitesimal width, then the energy of the second disc, $d T_{2}$, should depend on the energy of the previous disc as $d T_{2}=d T_{1} d \mathcal{L}_{e}\left(v_{1}, \Delta z\right)$, where $0 \leq \mathcal{L}_{e} \leq 1$ is the proportion of energy that is conserved after travelling a distance $\Delta z$ at a speed $v_{1}$, where in turn $v_{1}$ is the velocity of the first disc. Taking the above into consideration, the overall picture is that energy loss should be greater for greater velocities and travel distances, for each fluid disc element. 


\section{Damping with constant velocity}

The simplest model which reproduces the above qualitative behaviour is

$$
d \mathcal{L}_{e}(v, \Delta z)=\frac{1}{1+v \Delta z / H_{e}}=\frac{1}{1+\hat{v} \Delta \hat{z} / \hat{H}_{e}},
$$

where $H_{e}$ is some characteristic "energy half-loss" parameter at which half of the energy is lost for the given system, and $\hat{H}_{e}=H_{e} / v_{0} D_{0}=H_{e} / \nu_{0} \operatorname{Re}_{0}$, where $\nu_{0}$ and $\operatorname{Re}_{0}$ are the kinematic viscosity and Reynolds number of the liquid at the nozzle's exit, respectively. We can think of $H_{e}$ as the necessary distance traveled at a certain fixed speed for the fluid element to lose half its energy. Notice that $H_{e}$ has viscosity units, so we may consider it to be an energy dissipation parameter, similar to a turbulent viscosity. In this sense, the model hereof derived using (12) may be considered to include a simple turbulence model. Notice that, the greater the velocity $v$, the greater the energy loss; this is what we call "damping". The factor $d \mathcal{L}_{e}$ must be considered over the disc's whole trajectory to obtain the total loss. Consider a total flight distance $z$ from the nozzle exit until the target distance. Then there will be $z / \Delta z \equiv n$ total distance intervals and their corresponding time intervals, $\Delta t$, along the jet's trajectory. If the proportion of energy preserved after one such interval is $d \mathcal{L}_{e}$, then $\left(d \mathcal{L}_{e}\right)^{2}$ is the proportion of energy preserved after two such intervals, considering a constant speed $v_{0}$. Then the proportion of the initial energy preserved after travelling an axial distance $z$ from the nozzle exit is

$$
d \mathcal{L}_{e}^{n}=\left(\frac{1}{1+H_{e}^{-1} v_{0} z / n}\right)^{n}
$$

Proceeding to the infinitesimal limit when the disc width $\Delta z \rightarrow 0$, equivalent to $n \rightarrow \infty$, we get

$$
\lim _{n \rightarrow \infty} d \mathcal{L}_{e}^{n}=\lim _{n \rightarrow \infty} \frac{1}{\left(1+H_{e}^{-1} \frac{v_{0} z}{n}\right)^{n}}=\exp \left(-v_{0} z / H_{e}\right):=\mathcal{L}_{e}\left(v_{0}, z\right) .
$$

Using this function $\mathcal{L}_{e}(z)$ as a proportion factor we can rewrite our theory introducing energy loss as a function of axial distance from the nozzle exit. In dimensionless units, $\mathcal{L}_{e}(\hat{z})=\exp \left(-\hat{z} / \hat{H}_{e}\right)$. With this modification, (9) turns into

$$
\rho_{*} \hat{D}^{2} \hat{v}^{3}+\left(1-\rho_{*}\right) \hat{v}^{2}-\exp \left(-\hat{z} / \hat{H}_{e}\right)=0,
$$

and (10) into

$$
\left(\frac{\hat{\rho}-\rho_{*}}{1-\rho_{*}}\right)^{3}=\frac{\hat{\rho}}{\hat{D}^{4}} \exp \left(\hat{z} / \hat{H}_{e}\right) .
$$

We must remark that (13), (14), (15) and (16) hold approximately only for nearly constant axial speed along the jet's axis.

\section{Damping with varying velocity}

The assumption of constant axial speed proved to be fairly accurate when no energy loss factor like (12) was included. However, with the inclusion of energy loss, the velocity along the trajectory starts to considerably decrease with increasing energy loss, i.e. decreasing parameter $H_{e}$. This forces us to further rewrite the above equations into some recurrence formulas by which we can update the velocity value along the trajectory of the fluid element. This can be done by adapting (5) to depend on the immediately previous value. As for the density equation, it may be kept unmodified because the amount of liquid is calculated from the original state without gas entrainment and geometric considerations. Following a procedure analogous as in previous calculations, we obtain the velocity recurrence equation

$$
\rho_{g} v^{3}+\left(\frac{D_{0}}{D}\right)^{2} v_{0}\left(\rho_{0}-\rho_{g}\right) v^{2}-v_{i-1}^{3}\left(\frac{D_{i-1}}{D}\right)^{2} \frac{\rho_{i-1}}{1+v_{i-1} \Delta z H_{e}^{-1}}=0
$$

Notice that (17) reduces to (9) if $i=1$ and $H_{e} \rightarrow \infty$. For completeness, the equivalent of the non-linear equation for the density (10) as a recurrence equation is

$$
\left(\frac{\rho-\rho_{g}}{\rho_{0}-\rho_{g}}\right)^{3}=\frac{\rho v_{0}^{3} D_{0}^{6}\left(1+v_{i-1} \Delta z \gamma^{-1}\right)}{\rho_{i-1} v_{i-1}^{3} D_{i-1}^{2} D^{4}} .
$$

Notice that (18) also reduces to (10) if $i=1, v_{1}=v$ and $H_{e} \rightarrow \infty$.

\section{Mass loss}

We further generalize the above model and assume that only some proportion of the mass is conserved. We can identify the causes of mass loss as at least the following. (i) Escaping droplets: some droplets are expelled from the jet due to the breakup and atomization processes, taking with them mass. (ii) Evaporation: if the surrounding gas is not saturated in liquid vapour, evaporation will take place and, if this vapour escapes it will reduce the mass of the moving jet, albeit slowly. Both these causes also produce energy loss. The mass loss factors are affected by velocity and travel time/distance in the following ways. (i) Escaping droplets: droplets escape rate should be 
dependent on vorticity present and also on instability growth rates which produce jet breakup; both of these factors increase with velocity. (ii) Evaporation: may occur both from the jet's continuous part (if any) and from the droplets. Evaporation turns liquid into vapour which is taken away by the surrounding gas and eventually may escape the jet. For constant temperature and gas humidity, evaporation is "advection-driven" and depends also on the velocity. It is only natural then that the proportion of mass loss, due to at least the above listed factors, should depend on both the velocity and travel time/distance of each fluid element (disc).

\section{Mass loss with constant velocity}

Using an analogous loss factor $\mathcal{L}_{m}(z)$ we can rewrite our theory introducing mass loss. With this modification the velocity equation turns into

$$
\rho_{*} \hat{v}^{3}+\hat{D}^{-2}\left[\left(1-\rho_{*}\right) \exp \left(-\hat{z} / \hat{H}_{m}\right) \hat{v}^{2}-\exp \left(-\hat{z} / \hat{H}_{e}\right)\right]=0 .
$$

Notice that this reduces to the no mass loss case when $H_{m} \rightarrow \infty$. And the density equation turns into

$$
\left(\frac{\hat{\rho}-\rho_{*}}{1-\rho_{*}}\right)^{3}=\hat{\rho} \hat{D}^{-4} \exp \left[\hat{x}\left(\frac{1}{\hat{H}_{e}}-\frac{3}{\hat{H}_{m}}\right)\right] \text {. }
$$

Notice that this also reduces to the no mass loss case when $H_{m} \rightarrow \infty$. We must remark again that this calculation holds only for constant or nearly constant velocity along the jet flight.

\section{Mass loss with varying velocity}

As stated before, the assumption of constant velocity proved to be fairly accurate when no energy loss nor mass loss factors were included. However, with the inclusion of loss factors, the velocity along the trajectory starts to vary depending on the combination of the parameters, $H_{e}$ and $H_{m}$. This forces us to further rewrite the above equations into some recurrence formulas by which we can update the value of the velocity along the trajectory of the fluid element. This is done by adapting the equations to depend on an immediately previous value. Following a procedure analogous as in previous calculations, we obtain the velocity recurrence equation

$$
\rho_{*} \hat{v}_{i}^{3}+\left(\frac{\hat{D}_{i-1}}{\hat{D}_{i}}\right)^{2} \hat{v}_{i-1}\left(\frac{\hat{\rho}_{i-1}}{1+\hat{v}_{i-1} \Delta \hat{z} \hat{H}_{m}^{-1}}-\rho_{*}\right) \hat{v}_{i}^{2}-\hat{v}_{i-1}^{3}\left(\frac{\hat{D}_{i-1}}{\hat{D}_{i}}\right)^{2} \frac{\hat{\rho}_{i-1}}{1+\hat{v}_{i-1} \Delta \hat{z} \hat{H}_{e}^{-1}}=0 .
$$

Notice that (21) also reduces to (9) without loss factors if $H_{m}, H_{e} \rightarrow \infty$ and $i=1$. As for the density, we find that

$$
\left(\frac{\hat{\rho}_{i}-\rho_{*}}{\frac{\hat{\rho}_{i-1}}{1+\hat{v}_{i-1} \Delta \hat{z} \hat{H}_{m}^{-1}}-\rho_{*}}\right)^{3}=\quad\left(\frac{\hat{D}_{i-1}}{\hat{D}_{i}}\right)^{4} \frac{\hat{\rho}_{i}\left(1+\hat{v}_{i-1} \Delta \hat{z} \hat{H}_{e}^{-1}\right)}{\hat{\rho}_{i-1}} .
$$

Notice that (22) also reduces to (10) without any loss if $i=1$ and $H_{m}, H_{e} \rightarrow \infty$.

\section{Liquid-only mass loss}

In the previous modeling of the mass loss factor we implicitly considered mass loss to happen from both phases of the jet, i.e. losing mass from both liquid and gas. The latter is not realistic, since we expect that the mass-loss mechanisms affect only the liquid phase, as was described in the enlisted physical mechanisms above. An ambient gas does not lose any mass as it simply occupies any space not occupied by the liquid. Therefore, we may restrict the mass-loss to happen only at the liquid phase to get a different set of equations. First notice that the volume can be calculated as

$$
V_{j}^{g}=V_{j}-V_{j}^{l}=V_{j}-V_{j-1}^{l} d \mathcal{L}_{m}\left(v_{j-1}, \Delta z\right)
$$

where the superscripts "g" and "l" refer to the quantities for gas and liquid, respectively; and, if no superscript is written, the quantity refers to the composite two-phase fluid. Notice that $V_{j}^{l}$ cannot be calculated as the volume of a cylinder as with $V_{0}^{l}$ or $V_{j}$, since the shape of the distributed droplets inside the jet is irregular. However, we may calculate $V_{j}^{l}$ recursively, since we always know the total volume, $V_{j}=1 / 4 \pi D^{2} \Delta z$, we also know the initial liquid volume $V_{0}^{l}=1 / 4 \pi D_{0}^{2} \Delta z$ and the relationship $V_{j}^{l}=V_{j-1}^{l} d \mathcal{L}_{m}$. Now, separating mass loss to affect only the liquid phase we may calculate again the mean composite density as:

$$
\rho_{j}=\frac{\rho_{0} V_{j}^{l}+\rho_{g} V_{j}^{g}}{V_{j}},
$$

where the subscript " $j$ " indicates the " $j$-th step" position. We may write after simplification

$$
\rho_{j}=\rho_{g}+\frac{V_{j}^{l}}{V_{j}}\left(\rho_{0}-\rho_{g}\right)
$$

Notice that $\rho_{j}$ can be calculated directly from this formula using the previously calculated values of $V_{j}$ and $V_{j}^{l}$. 
As in the case when we introduced the energy loss with varying velocity, we should have the same equation for the velocity:

$$
v_{j}^{3}=\left(\frac{D_{j-1}}{D_{j}}\right)^{2} \frac{\rho_{j-1}}{\rho_{j}} v_{j-1}^{3} d \mathcal{L}_{e} .
$$

Eliminating $\rho_{j}$ from (24) and (25):

$$
v_{j}=\left(\frac{D_{j-1}}{D_{j}}\right)^{2 / 3}\left(\rho_{j-1} d \mathcal{L}_{e}\right)^{1 / 3} \rho_{j} v_{j-1}
$$

From (26) we can calculate $v_{j}$ directly having previously calculated $\rho_{j-1}$ and $\rho_{j}$. Notice that the velocity recurrence relation (26) can be written as

$$
v_{j}^{2}=\frac{\frac{1}{4} \pi D_{j-1}^{2} v_{j-1} d t \rho_{j-1}}{\frac{1}{4} \pi D_{j}^{2} v_{j} d t \rho_{j}} v_{j-1}^{2} d \mathcal{L}_{e}=\frac{V_{j-1} \rho_{j-1}}{V_{j} \rho_{j}} v_{j-1}^{2} d \mathcal{L}_{e}
$$

and then, rearranging terms,

$$
\frac{1}{2} V_{j} \rho_{j} v_{j}^{2}=\frac{1}{2} V_{v-1} \rho_{j-1} v_{j-1}^{2} d \mathcal{L}_{e}
$$

i.e. $K_{j}=K_{j-1} d \mathcal{L}_{e}$, which is just the kinetic energy conservation equation times the energy-loss factor. Both equations reduce to the original ones when $\Delta z \rightarrow 0$.

\section{Limiting and special cases}

Let us now calculate some special or limiting cases of the previous mathematical model. It is worth remembering for the following analysis that $\hat{D}=1+2 \hat{z} \tan \theta$ is the diameter of the jet.

An atomizing jet in a very thin atmosphere, $\rho_{g} \ll \rho_{0}$

With this assumption, which physically means a liquid jet inside a very thin atmosphere, from Equation (9) we see, after simplification, that

$$
v=\sqrt{\frac{2 p_{0}}{\rho_{0}}} \equiv v_{0},
$$

i.e. the velocity remains constant at the same initial value, which is consistent with the physical interpretation since the liquid droplets would not loose any energy to the surrounding vacuum since there would be no drag forces present. Now from Equation (10):

$$
\rho=\rho_{0}\left(\frac{D_{0}}{D_{0}+2 z \tan \theta}\right)^{2}
$$

where we can see that when $z \rightarrow 0$ then $\rho \rightarrow \rho_{0}$, accordingly since at the nozzle we have only liquid. As $z \rightarrow+\infty$ then $\rho \rightarrow 0$, the density of vacuum. Since in this special case both $v$ and $\rho$ are given in closed form from Equations (29) and (30), respectively, we can also calculate a closed form expression for the pressure from (11) as

$$
p=p_{0}\left(\frac{D_{0}}{D_{0}+2 z \tan \theta}\right)^{2} .
$$

The square factor in (31) is just the ratio of the diameters at the nozzle, $D_{0}$, and at the impact zone, $D=D_{0}+$ $2 z \tan \theta$, from where we can see that at the nozzle $p=p_{0}$ and $p \rightarrow 0$ as $z \rightarrow+\infty$.

The submerged jet, $\rho_{g}=\rho_{0}$

From (10) we have that $\rho=\rho_{g} \equiv \rho_{0}$, as expected so the density is just that of liquid for all distances. From (9) we get

$$
v=\left(\frac{D_{0}}{D_{0}+2 z \tan \theta}\right)^{2 / 3} v_{0}
$$

which still gives $v=v_{0}$ for $z=0$, i.e. at the nozzle exit, and $v \rightarrow 0$ as $z \rightarrow+\infty$, intuitively correct. Notice that in this case density remains constant and velocity decays with distance whilst for the case in vacuum we had the converse, viz. velocity remained constant and density decayed. While equation (32) appears to be inconsistent with the classical result for the velocity decay of a submerged jet, $z^{-1}$, e.g. [20, p. 150], the introduction of the energy loss factor as in (12) provides for adjusting of the decay to a faster rate. A similar approach as used in this paper by the same authors but imposing momentum conservation gave the well known result [9]. 


\title{
Comparison with experiments and discussion
}

Figure 2 shows the centreline velocity fits to experimental data for a single-phase air jet as reported in [9]. Each of the three curves represents the theoretical results of a different model. Both the continuous line and the dashed line belong to the ideal momentum jet model family reported in the aforementioned work. The dot-dashed line is given by the discrete model with liquid-only mass loss, for the particular case of the "submerged jet" (air jet) as considered in the corresponding experiment. We can thus see that this discrete model gives the closes fit among these models, increasing the precision, at the cost of losing the analytical solutions.

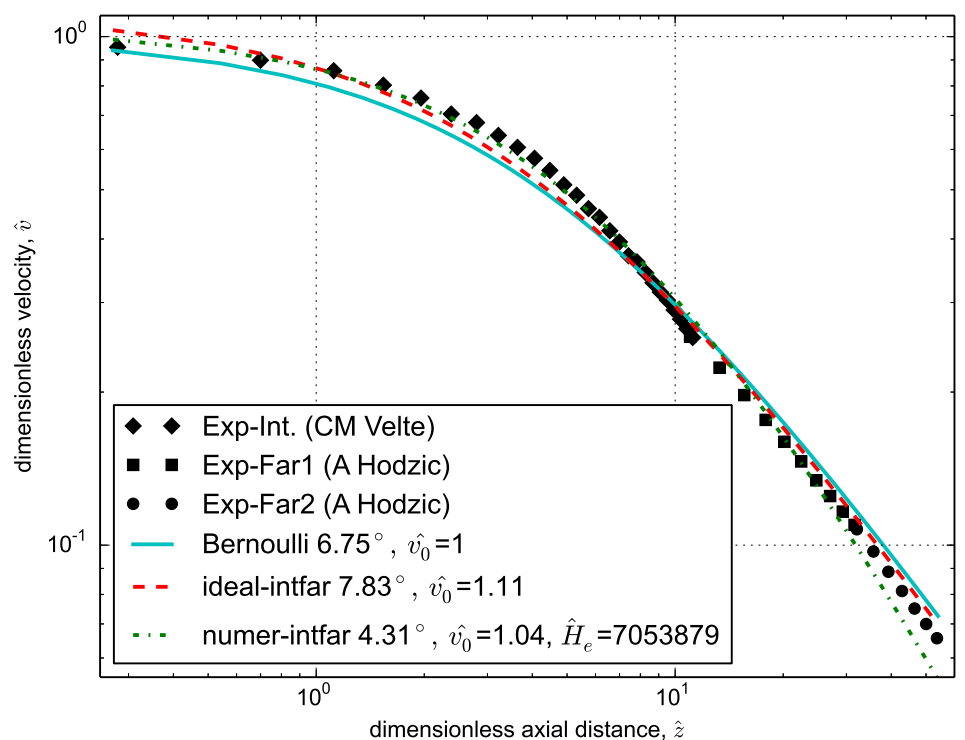

Figure 2. Centerline velocity fits to experimental data for a single-phase air jet as reported in [9].

\section{Conclusions}

We have presented three related one-dimensional mathematical models applicable to the dynamics of a wide class of turbulent atomizing jets. The models' main assumptions include the so-called Locally Homogeneous Flow (LHF) for a two-phase flow. The models are based on conservation laws of mass and energy, and describe the dynamical quantities, viz. density, velocity and dynamic pressure, along the jet's axis. The main advantages of the models over others in the literature are that the solutions can range from implicit to explicit analytical, that they contain a single main free parameter, viz. the jet's angle, and the fact that this angle can be approximated from experimental measurements with a method proposed in Reference [9]. We consider as special cases an atomizing liquid jet in a very thin atmosphere and a submerged jet. For the submerged jet, we carried out our own experiments with turbulent air jets, reported in [9], showing excellent agreement with the centerline velocity decay in the intermediate and far fields.

\section{Acknowledgements}

FFM was in part supported by the Mexican Council of Science and Technology (CONACYT), the Bank of Mexico (BANXICO)'s FIDERH program and KUMIAY Internacional, Co. Ltd.

\author{
Nomenclature \\ $z \quad$ Axial distance from the nozzle's exit \\ $D \quad$ Diameter of the jet \\ $\rho \quad$ Density \\ $m \quad$ mass \\ $v \quad$ Velocity \\ $p \quad$ Pressure \\ $\rho \quad$ Density \\ $\theta \quad$ Jet's cone apex angle \\ $H_{e} \quad$ Energy half loss parameter \\ $H_{m} \quad$ Mass half loss parameter \\ Subscripts and superscripts \\ $e \quad$ Entrainment or energy \\ 0 Quantity at the nozzle's exit position
}


$l \quad$ Liquid

$g \quad$ Gas

Dimensionless quantity

[1] Wakuri, Y., Fujii, M., Amitani, T., and Tsuneya, R., 1960. "Studies on the penetration of fuel spray in a diesel engine". Bull. Japan Soc. Mech. Eng., 3(900475), pp. 123-130. 1

[2] Sazhin, S. S., Feng, G., and Heikal, M. R., 2001. "A model for fuel spray penetration". Fuel, 80, pp. 2171-2180. 1,2

[3] Desantes, J. M., Payri, R., Salvador, F. J., and Gil, A., 2005. "Development and validation of a theoretical model for diesel spray penetration". Fuel, 85(2006), pp. 910-917. 1, 3

[4] Cossali, G. E., 2001. "An integral model for gas entrainment into full cone sprays". J. Fluid Mech., 439, pp. 353-366. 1

[5] Ghosh, S., and Hunt, J. C. R., 1994. "Induced air velocity within droplet driven sprays". Proc. R. Soc. London, Ser. A, 444(1920), Jan., pp. 105-127. 1, 3

[6] Jiang, X., Siamas, G. A., Jagus, K., and Karayiannis, T. G., 2010. "Physical modelling and advanced simulations of gas-liquid two-phase jet flows in atomization and sprays". Prog. Energy Combust. Sci., 36, pp. 131-167. 1

[7] Vallet, A., Burluka, A. A., and Borghi, R., 2001. "Development of an Eulerian model for the atomization of a liquid jet". Atomization and Sprays, 11(6). 1

[8] Stevenin, C., Vallet, A., Tomas, S., Amielh, M., and Anselmet, F., 2016. "Eulerian atomization modeling of a pressure-atomized spray for sprinkler irrigation". Int. J. Heat and Fluid Flow, 57, pp. 142-149. 1

[9] Franco Medrano, F., Fukumoto, Y., Velte, C. M., and Hodzic, A., 2017. "Mass entrainment rate of an ideal momentum turbulent round jet". J. Phys. Soc. Japan, 86(034401), mar. 2, 6, 7

[10] Franco Medrano, F., 2016. "Dynamics of a full-cone atomizing liquid jet". PhD thesis, Graduate School of Mathematics, Fukuoka, Japan, jun. 2

[11] Sazhin, S., 2006. "Multiple scales in spray modelling". J. Phys. Conf., 55, pp. 191-202. 2

[12] Fuchimoto, T., Yanase, S., Mizushima, J., and Senda, J., 2009. "Dynamics of vortex rings in the spray from a swirl injector". Fluid Dyn. Res., 41(4), May, p. 045503. 2

[13] Desantes, J. M., Salvador, F. J., López, J. J., and De La Morena, J., 2011. "Study of mass and momentum transfer in diesel sprays based on X-ray mass distribution measurements and on a theoretical derivation". Exp. Fluids, 2011(50), pp. 233-246. 2, 3

[14] Faeth, G. M., 1983. "Evaporation and Combustion of Sprays". Prog. Energy Combust. Sci., 9, pp. 1-76. 2

[15] Faeth, G. M., 1987. "Mixing, Transport and Combustion in Sprays". Prog. Energy Combust. Sci., 13, pp. 293345. 2

[16] Faeth, G. M., Hsiang, L.-P., and Wu, P.-K., 1995. "Structure and Breakup Properties of Sprays". Int. J. Multiphase Flows, 21, pp. 99-127. 2

[17] Rabadi, S. A., Friedel, L., and Surma, R., 2007. "Prediction of Droplet Velocities and Rain out in Horizontal Isothermal Free Jet Flows of Air and Viscous Liquid in Stagnant Ambient Air". Chem. Eng. Tech., 30(11), pp. 1546-1563. 3

[18] Pastor, J. V., López, J. J., García, J. M., and Pastor, J. M., 2008. "A 1D model for the description of mixingcontrolled inert diesel sprays". Fuel, 87(13), pp. 2871-2885. 3

[19] Drazin, P. G., and Reid, W. H., 2004. Hydrodynamic Stability, 2nd ed. Cambridge University Press.

[20] Landau, L. D., and Lifshitz, E. M., 1987. Fluid Mechanics, 2nd ed., Vol. 6 of Course of Theoretical Physics. Butterworth Heinemann. 6

[21] Vekstein, G. E., 1998. "Landau resonance mechanism for plasma and wind-generated water waves". Am. J. Physics, 66, p. 886. 